\title{
STOCHASTIC AND GEOMETRIC REASONING FOR INDOOR BUILDING MODELS WITH ELECTRIC INSTALLATIONS - BRIDGING THE GAP BETWEEN GIS AND BIM
}

\author{
Y. Dehbi ${ }^{\mathrm{a}, *}, \mathrm{~J}-\mathrm{H}$ Haunert ${ }^{\mathrm{a}, *}$ and L. Plümer ${ }^{\mathrm{a}}$ \\ ${ }^{a}$ Institute of Geodesy and Geoinformation, University of Bonn, Meckenheimer Allee 172, Bonn, Germany - \\ (dehbi, haunert, pluemer)@igg.uni-bonn.de
}

KEY WORDS: Stochastic reasoning, Constraint satisfaction, Gauss-Markov, Gaussian mixture, CityGML, BIM

\begin{abstract}
:
3D city and building models according to CityGML encode the geometry, represent the structure and model semantically relevant building parts such as doors, windows and balconies. Building information models support the building design, construction and the facility management. In contrast to CityGML, they include also objects which cannot be observed from the outside. The three dimensional indoor models characterize a missing link between both worlds. Their derivation, however, is expensive. The semantic automatic interpretation of 3D point clouds of indoor environments is a methodically demanding task. The data acquisition is costly and difficult. The laser scanners and image-based methods require the access to every room. Based on an approach which does not require an additional geometry acquisition of building indoors, we propose an attempt for filling the gaps between 3D building models and building information models. Based on sparse observations such as the building footprint and room areas, 3D indoor models are generated using combinatorial and stochastic reasoning. The derived models are expanded by a-priori not observable structures such as electric installation. Gaussian mixtures, linear and bi-linear constraints are used to represent the background knowledge and structural regularities. The derivation of hypothesised models is performed by stochastic reasoning using graphical models, Gauss-Markov models and MAP-estimators.
\end{abstract}

\section{MOTIVATION AND CONTEXT}

Building information models (BIMs) are widely used for building design, preconstruction analysis and construction planing. However, such models for as-built state are needed for a wide range of buildings. They are important and have widespread benefits for many tasks such as facility management. While BIMs for new constructions are manually designed based on various software, BIMs for existing buildings have to be derived from observations like $3 \mathrm{D}$ point clouds from laserscanners or range cameras. For the derivation of such models, especially for indoor environments, the necessary measurements are both cost and time extensive. In comparison to outdoor models where mainly airborne or terrestrial platforms are used for capturing data, the derivation of observations for indoor models is rather different. Every single room must be entered and scanned. Besides, the modelling of wall elements, doors, windows and ceilings turns out to be a difficult task, since they are often concealed by different kinds of furniture. Hence, it is necessary to identify and eliminate them from the model.

A BIM contains both semantically and geometrically rich information for the representation of the physical and functional features of a facility, however, surrounding information is not included (Rafiee et al. 2014). In contrast to BIM, a Geographic Information System (GIS) enables to perform spatial analysis incorporating the outdoor environment's functional and physical spatial relationships. Nevertheless, the contained building information is not rich enough for tasks such as building construction planning or facility management. In this context, the integration of building information and geospatial city models gains more and more attention. The goal is to benefit from the strengths of the two distinct worlds. In this manner, the monitoring and management of the progress in different levels of projects is enabled which supports decision making processes. Simultaneously, a spatio-temporal analysis can be performed in a wide range with respect to the project life cycle. (Liu et al. 2017). Especially, due to the increasing demand of digitalized models of existing build- ings, as-built building information modelling becomes an essential task. However, this task is facing various challenges such as the transition from observed building data to BIM objects as well as dealing with uncertainties characterising the data and the bilateral relations between the BIM substructures.

BIMs are further characterized by elements and substructures which cannot be observed from outside. This is, for example, the case of electric installations within the walls. For such objects, surveybased approaches do not constitute a relevant support. In this context, our motivation is to propose a concept and a method for deriving BIMs from sparse observations. In this paper, we demonstrate that 3D indoor models are derivable without the need of dense 3D observations. Especially geometric and stochastic reasoning are used in order to model regularities in building models taking the uncertainty of the underlying structures into account. In previous works, we derived successfully 2D (Loch-Dehbi and Plümer. 2011) and 3D (Dehbi et al. 2017a) façade models as well as 3D indoor models from sparse observations (Loch-Dehbi et al. 2017, Dehbi et al. 2017b). We believe that our methods can be transferred for the modelling of latent and not observable structures such as electric installations to enrich 3D building models by BIM relevant structures.

The remainder of this paper is structured as follows: Section 2 shows an overview on the related work. Section 3 gives insight into our previous works in the context of façade and indoor model generation. Building upon these methods, Section 4 presents a concept for the generation of BIMs using geometric and stochastic reasoning based on sparse observations. The paper is discussed and concluded in section 5

\section{RELATED WORK}

The creation of BIMs for new buildings during the planning and construction stage is a straightforward manual process. The derivation of as-built BIMs from surveying is however a still investi- 
gated research field. Nagel et al. (2009) analysed the requirements and the difficulties which an automatic reconstruction process is facing. They proposed a two-staged approach for BIM model reconstruction using CityGML (Gröger and Plümer. 2012) as an interface between 3D graphics models and BIM models. In this context, many works showed how to derive CityGML models from IFC models. Isikdag and Zlatanova (2009) proposed a formal framework for a semantic mapping between both models allowing for an automatic transformation. They showed that IFC models contain all necessary information to generate CityGML models in different levels of detail.

Conversely, the acquisition of BIMs from geospatial building models is a more demanding task. To this aim, surveying methods are needed for capturing 3D observations in a time consuming and expensive process. In this context, Xiong et al. (2013) presented an approach for the conversion of a 3D point cloud from a laser scanner for the creation of semantically rich 3D building models. To this end, the laser scanner has to be positioned at multiple locations for covering the whole targeted objects. The strengths of both BIM and GIS and especially the mutual benefits from their cooperation are described by De Laat and Van Berlo (2011). BIMs can serve as input for urban GIS models, in turn GIS can used for the design and integration of new buildings from a geospatial point of view. A literature review of works on BIM for existing buildings and future needs in this field is provided by Volk et al. (2014). Liu et al. (2017) categorized previous works on the integration of BIM and GIS into three levels consisting of data level, process level and application level and presented a state-of-the-art review on integrating both models.

\section{GENERATION OF FAÇADE AND INDOOR MODELS}

For the generation of building models, in previous works we follow a top-down approach believing that it is easier to verify or falsify hypotheses than to reconstruct models from observations in a bottom-up way. Therefore, different methods and structures for geometric and stochastic reasoning have been applied. We are confident that our paradigm is well-suited for enriching $3 \mathrm{D}$ (interior) building models with BIM structures. To this end, this section gives a short overview on these approaches in different applications.

For the derivation of 3D façade models, we used statistical relational learning, namely Markov Logic Networks (MLNs) (Richardson and Domingos, 2006) together with specific Support Vector Machines (SVMs) (Tsochantaridis et al. 2004) to learn and generate a weighted attributed context-free grammar (WACFG). MLNs enforce the topological and geometric constraints and address uncertainty explicitly providing probabilistic inference. Further, they are able to deal with partial observations, for example caused by occlusions. Besides, uncertain projective geometry (Heuel 2004) is used to deal with the uncertainty of the observations. The learned grammar has been applied to reconstruct façades from 3D point clouds. Figure 1 illustrates the model based reconstruction using the WACFG. For more details, the reader is referred to Dehbi et al. (2017a).

In another context, we proposed an approach which does not rely on $3 \mathrm{D}$ dense observations. In this work, we used constraint satisfaction methods, namely Constraint Logic Programming (CLP) (Gavanelli and Rossi. 2010) for solving a multi-linear problem. The constraints have been formulated as logical relations. The hypotheses search space is structured based on an appropriate representation of background knowledge, the definition of domains and constraints on façade model parameters and an intelligent combination of constraint propagation and stochastic rea-

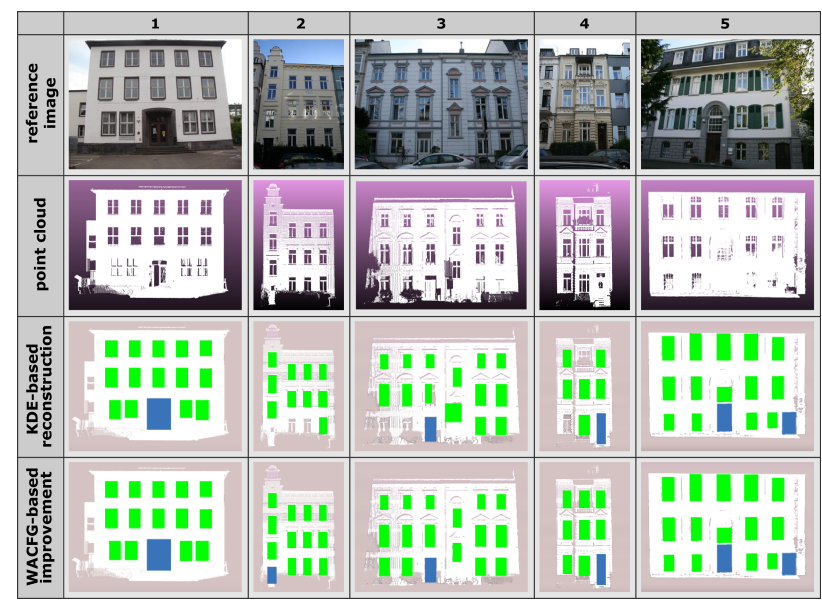

Figure 1: 3D façade reconstruction based on statistical relational learning (SRL) (Dehbi et al. 2017a).

soning yielding to solutions in a rather efficient way. The stochastic reasoning draws upon the combinatorial constraint satisfaction using special graphical models. Figure 2 shows some resulting ranked hypotheses based on an MAP-estimation. It should be noted that the observations consist only of the façade width and a single window embrasure. This approach makes use of an extensive data analysis leading to probability density functions (PDFs) of the related model parameters. PDFs of model parameters such as the distance between windows are represented by kernel density estimations (Wand and Jones 1994) or Gaussian mixtures as good approximations to model skew symmetric or multimodal distributions as illustrated by Figure 3 This enabled using well established reasoning algorithms. As stated in McLachlan and Peel (2000) each arbitrary probability density function can be approximated by Gaussian mixture models. More details to the applied method are provided by Loch-Dehbi and Plümer (2015).

Inspired by the method followed to predict façade models, LochDehbi et al. (2017) outlined an approach for the prediction of floorplans and indoor models without the need of indoor measurements. The algorithm profits from an extensive data analysis of shape and location parameters such as width and depth of rooms. This leads to a prior knowledge represented by architectural constraints and probability density functions. In turn, the considered constraints together with the PDFs approximated by Gaussian mixtures reduce the search space and enable to reconstruct floorplans based on otherwise insufficient data. In order to cope with the problem of exact inference in complex models, we combined Constraint Logic Programming with graphical models. The combinatorial problem of assigning each window to a room and determining the bilateral relations between rooms is solved by constraint propagation leading to preliminary topological models. This intermediate result is adjusted by a statistical component that aligns rooms along corridors where possible and estimates walls completing the floorplan model. Figure 4 gives an

\begin{tabular}{|c|c|c|c|c|c|c|c|}
\hline & AกIIIII & EI & $4 \times$ & $\sqrt{\mathrm{G}-\mathrm{A} A \mathrm{~A}}$ & E E & 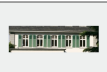 & पनत \\
\hline & [प्रा & 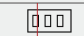 & 四司 & 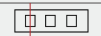 & प्रत्व & 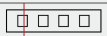 & पिक्ष \\
\hline & ए000 & [000 & DOQ & पिप्व & 回 & टि००० & \begin{tabular}{|l|l|} 
पC口 \\
\end{tabular} \\
\hline & पस्व & Fa & 䛛0 & पिण00 & 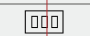 & 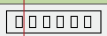 & पिस्व \\
\hline & [000000 & (4) & 四古 & 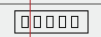 & (00000 & 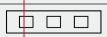 & 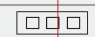 \\
\hline & 0 & एप्र & [व00000 & 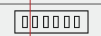 & ancono & 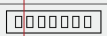 & 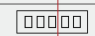 \\
\hline
\end{tabular}

Figure 2: Ranked façade hyptheses derived using constraint propagations and stochastic reasoning (Loch-Dehbi and Plümer 2015). 


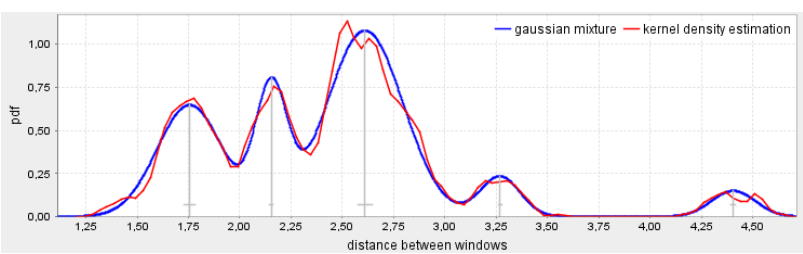

Figure 3: Gaussian mixture (blue) and kernel density estimation (red) for distance between two neighboring windows, mixture models good approximation with five means (gray vertical lines) and extremely small variances (gray horizontal lines). (LochDehbi and Plümer 2015)

overview on this approach and depicts the output and a resulting floorplan.

In Dehbi et al. (2017b), we focused on the improvement of model parameters in an a-priori estimated topological model that was generated by the use of Constraint Logic Programming as described in the last paragraph. Our approach makes use of a linear and non-linear Gauss-Markov model which incorporates not only probability density functions but also observations such as window locations or room areas. In order to achieve better accuracies, available symmetries have been exploited and their impact on the estimation has been investigated. Further symmetries in the known exterior model can be identified based on a classification task and subsequently modelled by formal grammars (Dehbi et al. 2016). Bayesian model selection is based on the information criteria AIC and BIC and incorporates observations in order to choose the hypotheses that best fit the scenario. Errors in the preliminary topological model are detected and corrected using a likelihood-based method. For more details the method is elaborated by Dehbi et al. (2017b). The 2D floorplans shown in Figure 5 represent different models derived taking different aspects such as symmetries into account.

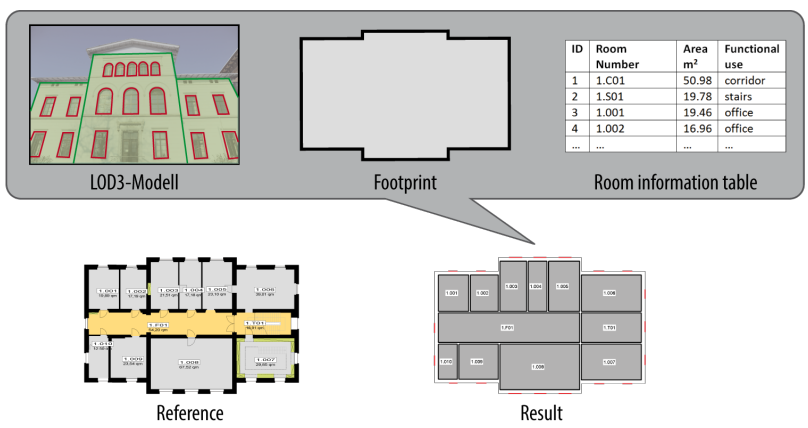

Figure 4: Automatic derivation of floor plans (bottom right) from sparse observations like window locations from possibly LoD3 exterior models, footprint and room information such as room areas (top). No additional indoor measurements are needed. For the comparison, a reference floor plan is depicted (bottom left). (Loch-Dehbi et al. 2017)

\section{GEOMETRIC AND STOCHASTIC REASONING FOR BIM MODELS}

The previous section demonstrated methods and approaches for the 3D building modelling and the automatic generation of semantic building models. In a similar spirit we are confident that the gaps between outdoor as well as indoor semantic modelling and building information modelling can be bridged. Especially, we believe that methods such as statistical relational learning, geometric and stochastic reasoning are adequate to tackle this task.
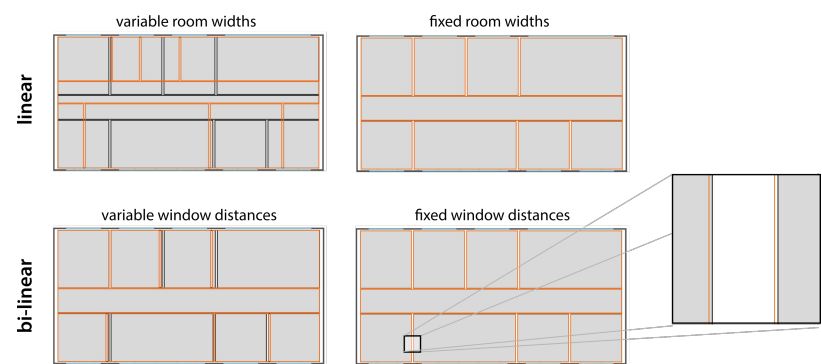

Figure 5: Floorplan estimation using a Gauss-Markov model. Model selection is performed based on linear (top) and bi-linear (bottom) constraints. Symmetries and repetitive patterns (fixed room widths, fixed window distances) are considered. The zoomed part on the right shows that the estimated (orange) and the ground truth model (black) are almost identical (Dehbi et al. 2017b)

Exemplarily, we discuss how to apply this mixture of methods to enrich 3D building models by further infrastructural information such as electric installations within existing buildings. Figure 6 represents a survey of our proposed concept illustrating the workflow of the different methods leading to a building model in LoD 4 which includes 3D indoor models and electric installations. In this context, we start from a 3D indoor model as shown in Figure 7 which has been derived by the reasoning process as described in section 3 We understand that man-made objects are characterized by a number of regularities. On the one hand, geometric relations such as parallelity and orthogonality are dominant in buildings. For instance, Steadman (2006) explained the predominance of the right angle in architectural plans. Loch-Dehbi and Plümer (2011) studied the geometric rules that can be found in man-made objects and presented an approach for deducing geometric relations in 3D building models. On the other hand, buildings can be described by functional and statistical dependencies between model parameters.

Likewise, regularities occur in non visible building elements. For example, in Germany the location of electric lines should follow some recommendations and have to respect underlying norms. There are two kinds of installation zones: vertical and horizontal zones. In order to avoid damaging of hidden lines, the locations of such lines are regulated. The horizontal installation zones are depicted in Figure 9 in blue colour. For the sake of safety, electric lines within this kind of zones have to be $30 \mathrm{~cm}$ away from the ceiling surfaces and likewise from the ground surface. This distance varies depending on the functional use of the according room. In rooms with working surfaces like countertops in kitchens, the distance amounts to $100 \mathrm{~cm}$. Power sockets are usually installed in the lower horizontal zones. Vertical installation zones highlighted in green colour are usually laid in a range of $15 \mathrm{~cm}$ from the windows (VZ-W), doors (VZ-d) and room edges (VZ-e). The recommended height for light switches is 105 $\mathrm{cm}$ from the ground surface. However, in special rooms such as kitchens this distance becomes $115 \mathrm{~cm}$. Otherwise, if the sockets or switches have to be inevitably installed outside the mentioned zones, the lines have to be laid in a vertical way from the next horizontal zone.

Background knowledge consisting of regularities and rules which characterize and regulate the electric infrastructures is analysed and exploited. The structure of the electric line network can be automatically learned from labelled data using statistical relational learning frameworks. Especially MLNs can be used in order to learn and model the geometric entities and the constraints among them. To this aim, logical facts as training data are derived from the spatial relational database (cf. Figure 8 for an MLN- 


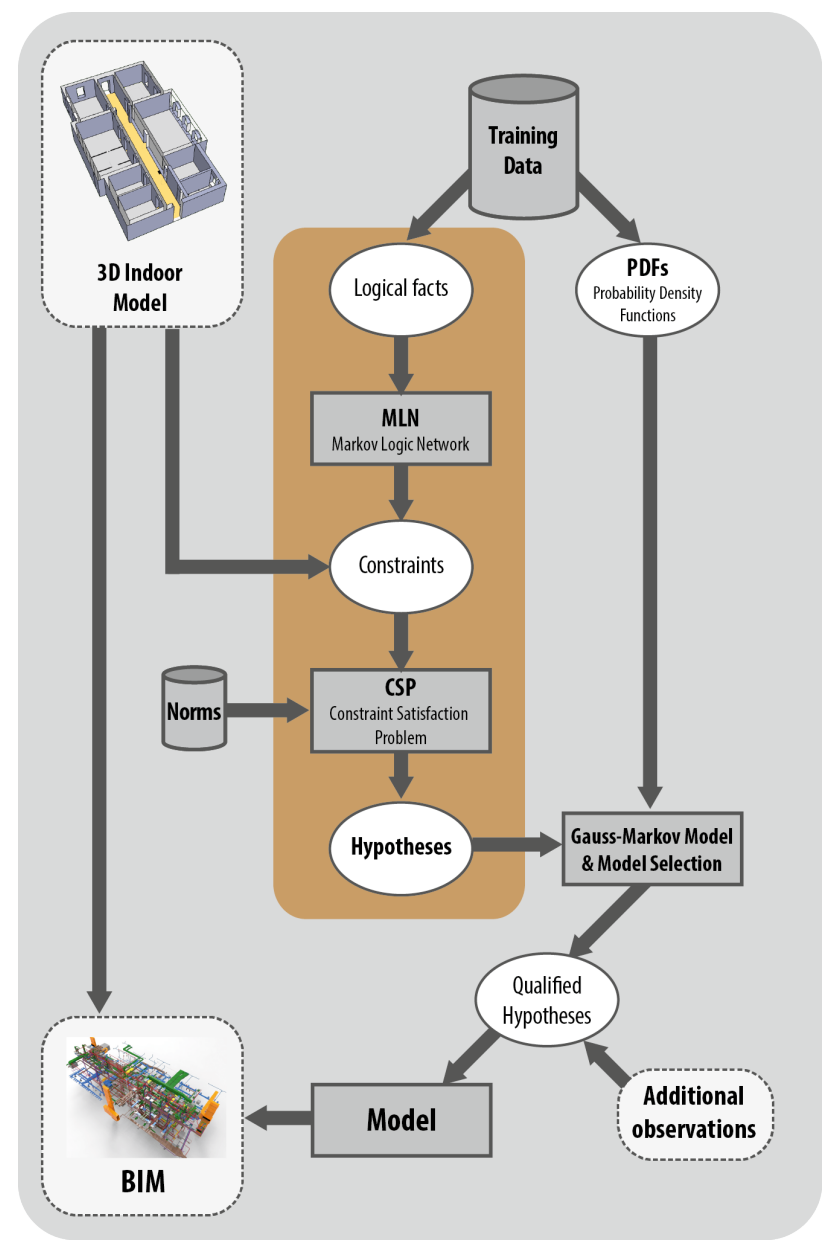

Figure 6: A survey of our concept for expanding 3D building models by electric installations for BIM.

based supervised learning task. The propagation of the constraints, especially between not neighboured objects, is ensured by statistical and relational inference as performed by Dehbi et al. (2017a). Inferred constraints and entities represent the input of a constraint satisfaction problem (CSP). In this manner, the rules can be formulated as constraints within a CSP. Besides, the background knowledge is expanded by probability density functions of location parameters. The latter depend on the building type and time. This reduces the hypotheses space. For the improvement of the hypotheses, Gauss-Markov models with a subsequent model selection can be used. Based on few additional observations, a transition from hypotheses into concrete models is achieved. The verification or falsification of such models can be performed with a low expense using appropriate sensors.

Despite the regularities derived from the recommendations, deviations from these expectations are not excluded. For this reason, an extensive data acquisition and a subsequent analysis is required. This enables the derivation of statistical knowledge in the form of probability density functions which can be approximated using Gaussian mixtures. Exemplarily, for façades, Figure 3 shows that the search space is structured by few peaks. The same paradigm has been also applied in Section 3 for the estimation of shape parameters of rooms. The probability distributions depend on the building style and building construction era. These parameters are incorporated in a conditional statistical reasoning process. Furthermore, the data analysis gives insight into the various location configurations of electric lines, switches and sockets independent from the mentioned recommendations.

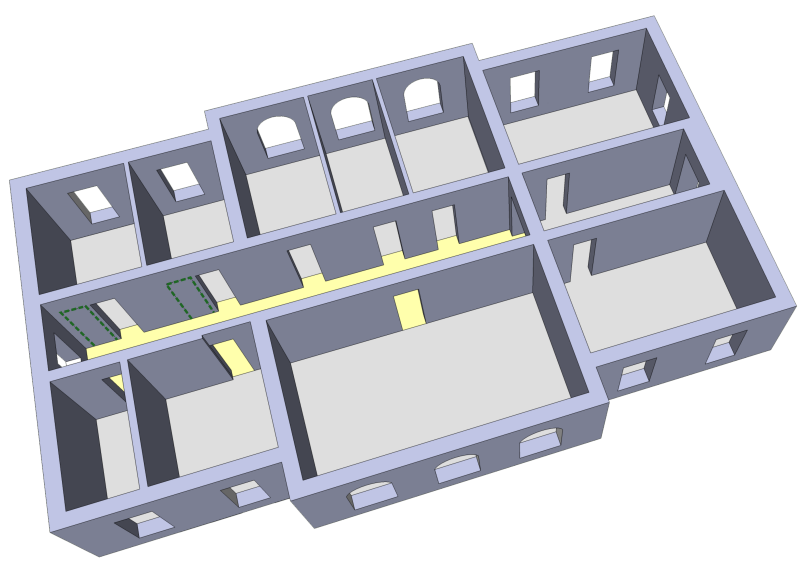

Figure 7: 3D indoor model resulting from an estimation process based on constraint propagation and stochastic reasoning (LochDehbi et al. 2017).

The data analysis and pattern recognition benefit from statistical and structural prior knowledge that is derived from a relational database from a previous project of about 1600 rooms with different functional uses. In order to model electrical infrastructure, the database is being extended by spatial information and new relevant objects and their relationships. Figure 8 shows a relevant excerpt of the underlying expanded database model. The relation room is still central to our analysis with shape and location parameters of each room as well as its functional use. Rooms reference their corresponding flats, which enables access to available footprints from several floors belonging to a given building. The relation building gives insight into information such as construction year and building style, which provides a beneficial prior for detecting contextual patterns. The location of windows and doors are modelled using the relations window and door respectively. The latter relation reveals information about accessibility between rooms. The neighbourhood of rooms is annotated in order to analyse the bilateral locations e.g., with respect to the functional use of rooms enabling to model and detect possible shared infrastructures. The relation devicetype refers to different types of electrical devices such as switches, sockets or intercoms. Devices themselves are modelled by the relation device. Mutual relations between devices are captured in order to detect possible symmetries and regularities supporting the prediction of occluded objects later on. The devices are associated to vertices that are end or intersection points using the relation vertex. Electric lines connecting vertices are represented by the relation powerline. This structure enables an eventual topological and geometrical analysis.

Note that this data does not serve as direct input for the reasoning process but is a representative basis for the derivation of probability distributions and structural constraints for the spatial enriched BIM model and its prediction. For the annotation of the electric lines we are testing the usefulness of thermography for the detection of not visible lines. In this context, the infra-red camera VarioCAM HD research 90 is used as sensor with a measurement accuracy of $\pm 1^{\circ} \mathrm{C}$ and a temperature resolution of $0.02 \mathrm{~K}$.

Inspired by the approaches for façade as well as indoor model prediction, geometric and stochastic reasoning can be used to predict switches occluded by furnitures. Symmetry information and statistical inference turn out to be an adequate tool to deal with this issue. Single observations can be added to assess the quality of hypotheses. Figure 10 illustrates likewise the impact of a further observed embrasure on the certainty of façade hypotheses. In this context, another interesting question is to guess which kind 


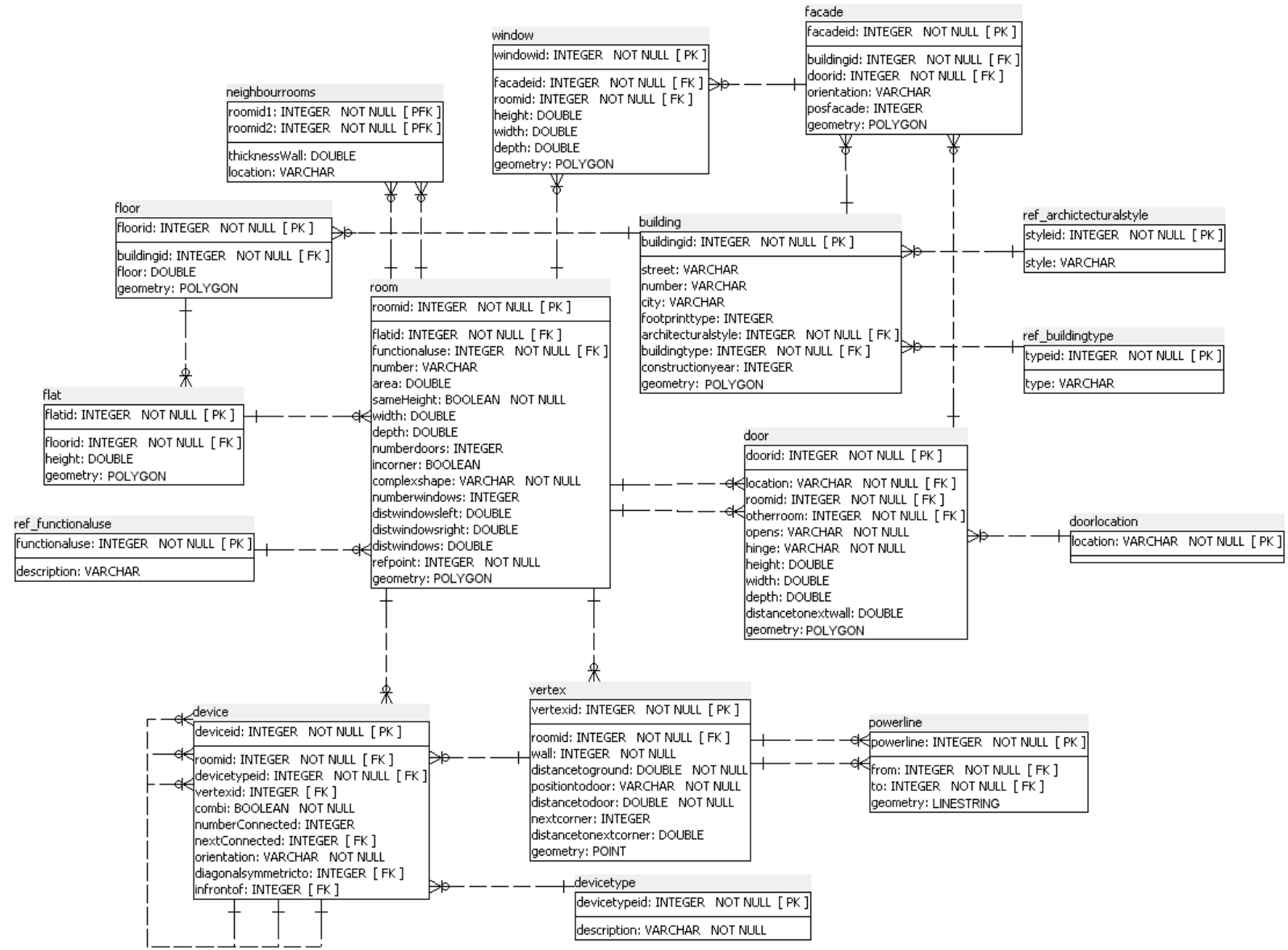

Figure 8: Excerpt from the relational database schema. Spatial objects, e.g. power line, reveal location and shape parameters. The database is used for training and evaluation.

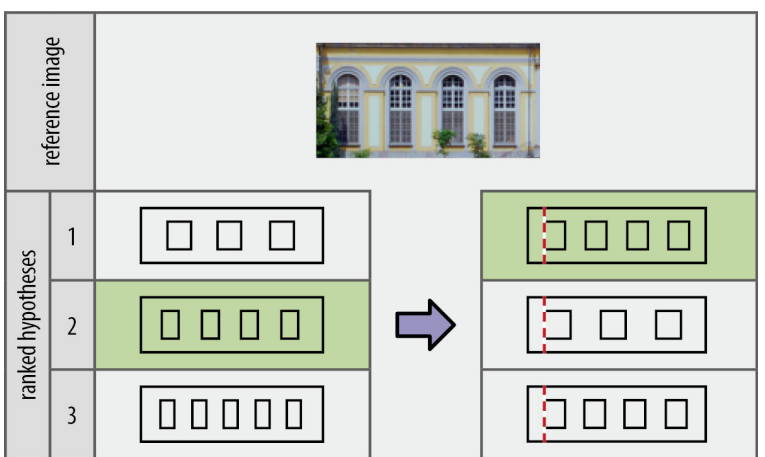

Figure 10: The impact of additional observations on the certainty of model hypotheses. Certainty is increased by considering a single observed embrasure (Loch-Dehbi and Plümer, 2015). This approach can be adapted for assessing and improving the quality of electric line hypotheses.

of additional observations can be taken into consideration for the validation of hypotheses.

As stated, the installation paths of the electric lines are usually following some regular patterns. In analogy to regularities such as parallelities, orthogonalities and symmetries characterising building models, the structure of these installations can be modelled as a graph $G=(V, E)$ consisting of vertices $V$ (switches, sockets and intersection points) and edges $E$ (line segments). In order to ensure a valid structure, a constraint satisfaction problem can be solved (Dechter. 2003) similar to the approaches described in Loch-Dehbi and Plümer (2015) and Loch-Dehbi et al. (2017) for the prediction of façade and indoor models (cf. Section 3). In order to deal with uncertain observations and perform geometric reasoning over imprecise objects such electric lines and their mutual relations, uncertain projective geometry (Heuel, 2004) turns out to be an adequate tool. According to Figure 9 , geometric relations consist mainly of orthogonality and parallelity of lines. In this context, geometric entities like lines are augmented by covariance matrices enabling to make decisions about bilateral relations between these entities using a hypothesis test as performed in Dehbi and Plümer (2011) and Dehbi et al. (2017a).

To sum up, we have shown how to automatically derive 3D building models from observations such as 3D point clouds and also merely based on sparse observations. The combination of Gaussian mixtures, bi-linear constraints, constraint propagation, stochastic and relational reasoning turns out to be a powerful tool with regard to 3D building models. We have discussed how to apply this mixture of methods to enrich 3D building models by additional BIM related information. We have demonstrated the feasibility in a use case for the generation of hypotheses to localize the electric installations. The hypotheses are derived based on statistical inference by the use of Gaussian mixtures, Gauss-Markov models and an MAP-estimation.

\section{CONCLUSION}

This paper gave insight into successfully applied methods for the automatic derivation of 3D building models, especially based on weak observations. In this work, we have demonstrated that building upon these methods and previous experiences for the 


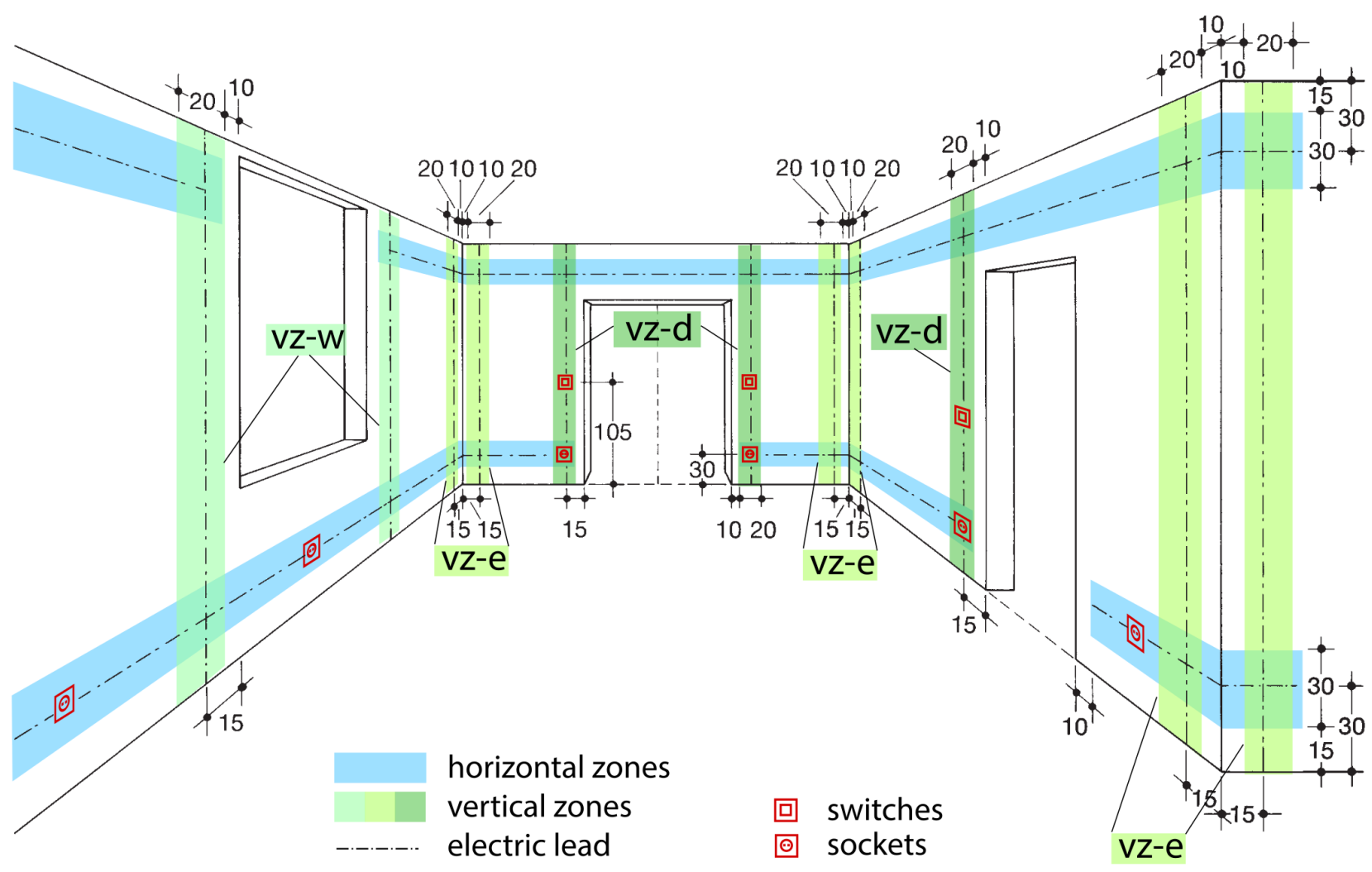

Figure 9: Recommended zones for electric lines according to the standard DIN 18015-3 in Germany. Horizontal zones (HZ) are highlighted in blue and vertical zones (VZ) in green colour. Electric leads are depicted as dotted lines. Preferred locations of power sockets and light switches are graphically depicted.

automatic derivation of 3D building models, building information modelling (BIM) can be performed to derive as-built models without the need of dense 3D observations. Methods such as Gaussian mixtures, bi-linear constraints, constraint propagation, stochastic and relational reasoning turn out to be powerful tools for dealing with this issue.

Exemplarily, we discuss how to apply this mixture of methods to enrich 3D building models by further infrastructural information such as electric installations within existing buildings. In this context, background knowledge consisting of regularities and rules which characterize and regulate these infrastructures is analysed and exploited. The rules can be formulated as constraints within a constraint satisfaction problem. Besides, the background knowledge is expanded by probability density functions of location parameters. The latter depend on the building type and time. This reduces the hypotheses space and enables the transition from hypotheses into concrete models based on few additional observations. The verification or falsification of such models is performed with a low expense using appropriate sensors. Hypotheses for the location of electric installation are derived based on statistical inference using Gaussian mixtures and an MAP-estimation.

The presented concept is not restricted to electrical lines. It can be extended in order to predict other types of infrastructure such as communication and water facilities. This is a first step in a large project for enriching 3D building models leading to expanded building information models.

\section{ACKNOWLEDGEMENTS}

We thank Sandra Loch-Dehbi for the valuable cooperation. The authors are grateful to Stefan Teutsch for his assistance in preparing the illustrations.

\section{REFERENCES}

De Laat, R. and Van Berlo, L., 2011. Integration of BIM and GIS: The development of the CityGML GeoBIM extension. In: Advances in 3D geo-information sciences, Springer, pp. 211-225.

Dechter, R., 2003. Constraint processing. Elsevier Morgan Kaufmann.

Dehbi, Y. and Plümer, L., 2011. Learning grammar rules of building parts from precise models and noisy observations. ISPRS Journal of Photogrammetry \& Remote Sensing 66(2), pp. 166176.

Dehbi, Y., Gröger, G. and Plümer, L., 2016. Identification and modelling of translational and axial symmetries and their hierarchical structures in building footprints by formal grammars. Transactions in GIS 20(5), pp. 645-663.

Dehbi, Y., Hadiji, F., Gröger, G., Kersting, K. and Plümer, L., 2017a. Statistical relational learning of grammar rules for $3 \mathrm{~d}$ building reconstruction. Transactions in GIS 21(1), pp. 134-150.

Dehbi, Y., Loch-Dehbi, S. and Plümer, L., 2017b. Parameter estimation and model selection for indoor models based on sparse observations. Accepted for the Indoor3D Workshop, Geospatial week, Wuhan, China. 
Gavanelli, M. and Rossi, F., 2010. Constraint logic programming. in a 25 -year perspective on logic programming. In: erspective on Logic Programming, Vol. 6125, pp. 64-86.

Gröger, G. and Plümer, L., 2012. CityGML-interoperable semantic $3 \mathrm{~d}$ city models. ISPRS Journal of Photogrammetry and Remote Sensing 71, pp. 12-33.

Heuel, S., 2004. Uncertain projective geometry: Statistical reasoning for polyhedral object reconstruction. Springer, Berlin.

Isikdag, U. and Zlatanova, S., 2009. Towards defining a framework for automatic generation of buildings in citygml using building information models. In: 3D geo-information sciences, Springer, pp. 79-96.

Liu, X., Wang, X., Wright, G., Cheng, J. C., Li, X. and Liu, R., 2017. A state-of-the-art review on the integration of building information modeling (bim) and geographic information system (gis). ISPRS International Journal of Geo-Information 6(2), pp. 53.

Loch-Dehbi, S. and Plümer, L., 2011. Automatic reasoning for geometric constraints in $3 \mathrm{~d}$ city models with uncertain observations. ISPRS Journal of Photogrammetry and Remote Sensing 66(2), pp. 177-187.

Loch-Dehbi, S. and Plümer, L., 2015. Predicting building facade structures with multilinear gaussian graphical models based on few observations. Computer, Environment and Urban Systems 54, pp. 68-81.

Loch-Dehbi, S., Dehbi, Y. and Plümer, L., 2017. Estimation of 3d indoor models with constraint propagation and stochastic reasoning in the absence of indoor measurements. ISPRS International Journal of Geo-Information.

McLachlan, G. and Peel, D., 2000. Finite Mixture Models. Wiley series in probability and statistics: Applied probability and statistics, Wiley.

Nagel, C., Stadler, A. and Kolbe, T. H., 2009. Conceptual requirements for the automatic reconstruction of building information models from uninterpreted $3 \mathrm{~d}$ models. International Archives of Photogrammetry, Remote Sensing and Spatial Information Sciences 38 (34/C3) pp. 46-53.

Rafiee, A., Dias, E., Fruijtier, S. and Scholten, H., 2014. From bim to geo-analysis: View coverage and shadow analysis by bim/gis integration. Procedia Environmental Sciences 22, pp. 397 - 402. 12th International Conference on Design and Decision Support Systems in Architecture and Urban Planning, DDSS 2014.

Richardson, M. and Domingos, P., 2006. Markov logic networks. Mach. Learn. 62(1-2), pp. 107-136.

Steadman, P., 2006. Why are most buildings rectangular? Architectural Research Quarterly 10(02), pp. 119-130.

Tsochantaridis, I., Hofmann, T., Joachims, T. and Altun, Y., 2004. Support vector machine learning for interdependent and structured output spaces. In: International Conference on Machine Learning (ICML), pp. 104-112.

Volk, R., Stengel, J. and Schultmann, F., 2014. Building information modeling (bim) for existing buildings literature review and future needs. Automation in Construction 38, pp. $109-127$.

Wand, M. P. and Jones, M. C., 1994. Kernel smoothing. Crc Press.
Xiong, X., Adan, A., Akinci, B. and Huber, D., 2013. Automatic creation of semantically rich $3 \mathrm{~d}$ building models from laser scanner data. Automation in Construction 31, pp. 325-337. 